\title{
Hydrofluoric acid burn
}

\author{
Siyu Wang MD PhD, Gengwu Dai MD
}

Cite as: CMAJ 2019 March 18;191:E314. doi: 10.1503/cmaj.181078

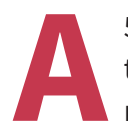

52-year-old man presented to the outpatient department with severe pain, right palmar erythema and blanching for 3 days, and necrosis on the tips of 2 fingers for 1 day. Three days earlier, the patient had worn cotton gloves to twist off the cap of a bottle of $40 \%$ hydrofluoric acid to prepare a solution for crop pest control. At first, erythematous patches developed with minor discomfort, and he delayed first aid treatment, including water rinsing. Several hours later, grey patches and worsening pain developed, leading to progressive necrosis of his fingers (Figure 1A).

Results from physical and laboratory tests were unremarkable, except for a slight reduction in his serum calcium level (2.03 [normal range 2.25-2.75] mmol/L). Histopathology of 1 of the skin lesions showed poorly defined tissue structures of the fulllayer dermis owing to severe liquefactive necrosis (Figure 1B). We diagnosed burns caused by contact with hydrofluoric acid. Surgical débridement and soaking of the hand with a calcium solution relieved the excruciating pain. The patient had recovered well without any residual damage 1 month later, accompanied by the loss of 2 finger tips after the surgical débridement.

Hydrofluoric acid is a dangerous inorganic acid that is widely used in electronics manufacturing, glass etching, removal of rust, pest control in agriculture and heavy-duty domestic cleaning. ${ }^{1}$ The fluoride ions in hydrofluoric acid are strong scavengers of bivalent cations, such as calcium and magnesium, and can slowly penetrate skin tissue, resulting in necrosis. Many workers using this acid may not be aware of the hazards and protective measures that are needed.

Early symptoms of hydrofluoric acid burn can be insidious at concentrations of less than 50\%, and reactions to the acid may be confused with allergic contact dermatitis. Accurate diagnosis and timely management of burns caused by hydrofluoric acid are crucial. Failure to recognize this type of burn may lead to irreversible local tissue necrosis, systemic poisoning (hyperkalemia, hypocalcemia and hypomagnesemia) and even death. ${ }^{2}$
The critical procedures in treating burns caused by hydrofluoric acid are prevention of absorption of the acid and blocking the progressive destruction caused by fluoride ions. The mainstays of treatment are early water irrigation, topical application of calcium gluconate reagents and surgical intervention.,

\section{References}

1. Stuke LE, Arnoldo BD, Hunt JL, et al. Hydrofluoric acid burns: a 15-year experience. J Burn Care Res 2008;29:893-6.

2. Ohtani M, Nishida N, Chiba T, et al. Pathological demonstration of rapid involvement into the subcutaneous tissue in a case of fatal hydrofluoric acid burns. Forensic Sci Int 2007;167:49-52.

3. Wang X, Zhang Y, Ni L, et al. A review of treatment strategies for hydrofluoric acid burns: current status and future prospects. Burns 2014;40:1447-57.

4. Alper N, Desai K, Rabinowitz S. Management of hydrofluoric acid burns. Eplasty 2014;14:ic42.

\section{Competing interests: None declared.}

This article has been peer reviewed.

The authors have obtained patient consent.

Affiliation: Department of Dermatology, Institute of Dermatology and Venereology, Sichuan Academy of Medical Sciences and Sichuan Provincial People's Hospital, Chengdu, Sichuan, China

Correspondence to: Siyu Wang, graceyuyu@126.com 